DOI: 10.32370/IAJ.2549

\title{
"Doppler de-boosting" and the observation of "Standard candles" in cosmology
}

M. Zilberman ${ }^{1}$

\begin{abstract}
"Doppler boosting" is a well-known relativistic effect that alters the apparent luminosity of approaching radiation sources. "Doppler de-boosting" is the name of relativistic effect observed for receding light sources (e.g. relativistic jets of active galactic nuclei and gamma-ray bursts). "Doppler boosting" changes the apparent luminosity of approaching light sources to appear brighter, while "Doppler de-boosting" causes the apparent luminosity of receding light sources to appear fainter. While "Doppler de-boosting" has been successfully accounted for and observed in relativistic jets of AGN, it was ignored in the establishment of Standard candles for cosmological distances. A Standard candle adjustment of an $\mathrm{Z}>0.1$ is necessary for "Doppler de-boosting", otherwise we would incorrectly assume that Standard Candles appear dimmer not because of "Doppler deboosting" but because of the excessive distance, which would affect the entire Standard Candles ladder at cosmological distances. The ratio between apparent $(\mathrm{L})$ and intrinsic $\left(\mathrm{L}_{\mathrm{o}}\right)$ luminosities as a function of the redshift $\mathrm{Z}$ and spectral index $\alpha$ is given by the formula $\mathcal{M}(\mathrm{Z})=\mathrm{L} / \mathrm{L}_{0}=(\mathrm{Z}+1)^{\alpha-3}$ and for Type Ia supernova appears as $\mathcal{M}(\mathrm{Z})=\mathrm{L} / \mathrm{L}_{0}=(\mathrm{Z}+1)^{-2}$. "Doppler de-boosting" may also explain the anomalously low luminosity of objects with a high $\mathrm{Z}$ without the introduction of an accelerated expansion of the Universe and Dark Energy.
\end{abstract}

\section{"Doppler boosting" and "Doppler de-boosting"}

"Doppler boosting" is a well-known relativistic effect that increases the apparent luminosity of approaching light sources. Specifically, it allows to obtain the intrinsic value of the luminosities $\left(\mathrm{L}_{\mathrm{o}}\right)$ of relativistic jets by their apparent luminosity $(\mathrm{L})$, velocities and the spectral index [1], [2] (also [3] for LS I $+61^{0} 303$.) This relationship is described by the following formulas

$$
\mathrm{L}=\mathrm{L}_{0} \delta^{\mathrm{p}}
$$

where the Doppler factor $\delta$ is

$$
\delta=\gamma^{-1}(1-\beta \cos \theta)^{-1}
$$

the Lorentz factor $\gamma$ is

$$
\gamma=\left(1-\beta^{2}\right)^{-1 / 2}
$$

the velocity $\beta$ is the speed $V$ of a relativistic light source normalized to the speed of light $C$

$$
\beta=v / c
$$

$\theta$ is the angle between line of sight and the velocity direction, $\alpha$ is the spectral index $\left(S_{\nu} \propto \nu^{\alpha}\right)$ and $\mathrm{p}=2-\alpha$ for a continuous jet emission, and $\mathrm{p}=3-\alpha$ for a discrete emitting region [2].

"Doppler de-boosting" [4], [5] is a nickname of the same relativistic effect calculated and observed for receding sources of radiation, for example for receding jets of active galactic

1. Shiny World Corporation, Concord, L4K 0C0, Canada. Email: editor@ IntellectualArchive.com 
nuclei (AGN). While "Doppler boosting" alters the apparent luminosity of approaching $\left(0<=\theta<=90^{\circ}\right)$ sources to be greater, "Doppler de-boosting" alters the apparent luminosity of the receding $\left(90^{\circ}<\theta<=180^{\circ}\right)$ sources to be fainter. (In [6] "Doppler de-boosting" named as the "Dimming effect" was obtained by the calculation of the number of photons arriving from receding light sources).

\section{"Doppler de-boosting" and "Standard candles"}

Even if "Doppler de-boosting" was successfully observed in relativistic jets of the AGN and gamma-ray bursts (GRBs), it was not accounted for in the establishment of Standard candles for cosmological distances. Although disregarding "Doppler de-boosting" for low-speed Standard candles simplifies calculations, for Standard candles with $\mathrm{Z}>0.1$ the correction of "Doppler de-boosting" appears to be necessary. Otherwise, we will incorrectly assume that Standard candles appear dimmer not because of "Doppler de-boosting" but because they are located further away than in actuality. This will affect the entire idea of the Standard candles ladder at cosmological distances and the following cosmological models.

The following presents "Doppler de-boosting" using the redshift parameter $\mathrm{Z}$ used in Cosmology.

As for cosmological objects with the angle $\theta=180^{\circ}$, the Doppler factor $\delta$ becomes

$$
\delta=\gamma^{-1}(1+\beta)^{-1} \equiv\left(1-V^{2} / c^{2}\right)^{1 / 2}(1+v / c)^{-1}
$$

And since the redshift parameter $\mathrm{Z}$ and velocity $V$ are linked as

$$
\mathrm{Z}+1=(1+v / \mathrm{c})\left(1-V^{2} / \mathrm{c}^{2}\right)^{-1 / 2}
$$

the $\delta$ and $\mathrm{Z}$ are linked as

$$
\delta=1 /(\mathrm{Z}+1)
$$

Since for discrete emitting region parameter $p=3-\alpha[2]$, the apparent luminosities (L), redshift parameter $\mathrm{Z}$ and the intrinsic luminosities $\left(\mathrm{L}_{0}\right)$ relation is shown as

or

$$
\begin{gathered}
\mathrm{L}=\mathrm{L}_{\mathrm{o}} /(\mathrm{Z}+1)^{3-\alpha} \equiv \mathrm{L}_{\mathrm{o}}(\mathrm{Z}+1)^{\alpha-3} \\
\mathcal{M}(\mathrm{Z}) \equiv \mathrm{L} / \mathrm{L}_{\mathrm{o}}=(\mathrm{Z}+1)^{\alpha-3}
\end{gathered}
$$

where $\mathcal{M}(Z)$ is the ratio between the apparent and intrinsic luminosities as a function of redshift $\mathrm{Z}$ and spectral index $\alpha$.

Regarding spectral index $\alpha$ in equation (9). For Type Ia supernova (SNIa), which are considered as Standard Candles on cosmological distances, per [7] the spectral index $\alpha$ is about 1 (by "Joint Light-curve Analysis" sample containing 740 SNIa data points) and for the supernova remnant RCW 86 the spectral index is between 1.5 and 2 [8]. In other words, for SNIa we can expect a relationship between $L$ and $L_{o}$ as 


$$
\mathcal{M}(\mathrm{Z}) \equiv \mathrm{L} / \mathrm{L}_{0}=(\mathrm{Z}+1)^{-2}
$$

Chart fig. 1 presents the influence of Doppler de-boosting to the apparent luminosities of SNIa for spectral indices $\alpha=1$ and $\alpha=2$, i.e. $\mathcal{M}(\mathrm{Z})=(\mathrm{Z}+1)^{-2}$ and $\mathcal{M}(\mathrm{Z})=(\mathrm{Z}+1)^{-1}$.

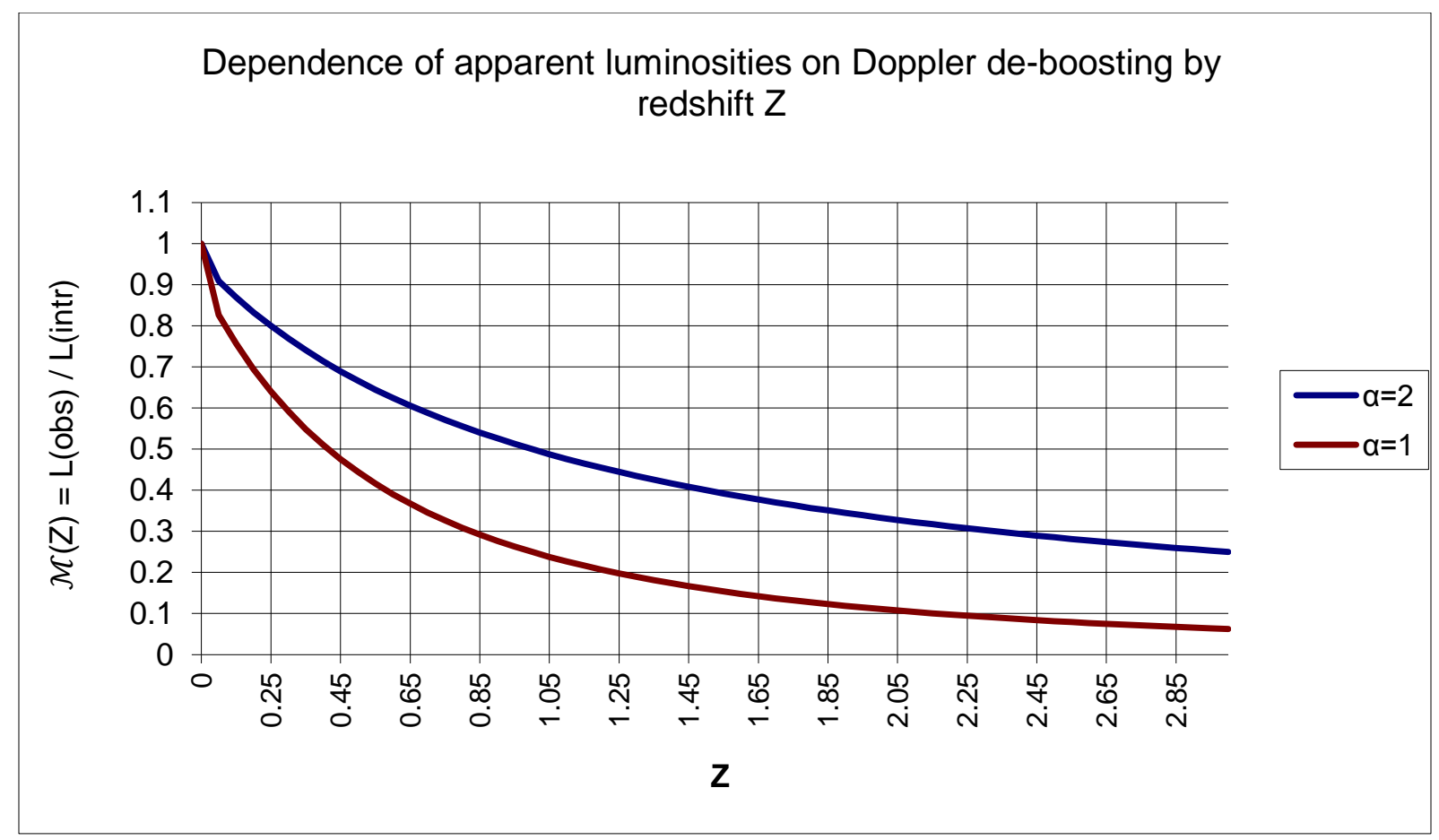

Fig.1. Influence of Doppler de-boosting to the apparent luminosities of SNIa for spectral indexes $\alpha=1$ (red line) and $\alpha=2$ (blue line).

As we see, the luminosity of objects receding from the observer with a redshift of $Z=3$ appears 4 times fainter for spectral index $\alpha=2$ and appears 16 times fainter for spectral index $\alpha=1$. If we do not consider the "Doppler de-boosting" effect, we can incorrectly assume that these objects are located many times further than they truly are.

Hence, the "Doppler de-boosting" effect (which is already confirmed in relativistic jets of GRB and AGN) may possibly explain the anomalously low luminosity of objects with a high $\mathrm{Z}$ without the introduction of an accelerated expansion of the Universe and following, the introduction of Dark energy.

\section{References}

1. Kellermann, K.I., Kovalev, Y.Y., Lister, M.L. et al. Doppler boosting, superluminal motion, and the kinematics of AGN jets. Astrophys Space Sci 311, 231-239 (2007). https://doi.org/10.1007/s10509-007-9622-5

2. M. L. Lister. Altered luminosity functions of relativistically beamed jet populations, The ApJ, Volume 599:105-115, (2003). https://iopscience.iop.org/article/10.1086/379241 
3. M. Massi, G. Torricelli-Ciamponi. Intrinsic physical properties and Doppler boosting effects in LS I $+61^{0} 303$. A\&A 564, A23 (2014), https://doi.org/10.1051/00046361/201322760.

4. Zhou, J., \& Su, Y. (2006). Doppler boosting and de-boosting effects in relativistic jets of AGNs and GRBs. Proceedings of the International Astronomical Union, 2(S238), 477-478. https://doi.org/10.1017/S1743921307006035

5. J. Yang, C. Brocksopp, S. Corbel, Z. Paragi, T. Tzioumis, R. P. Fender. A decelerating jet observed by the EVN and VLBA in the X-ray transient XTE J1752-223, Monthly Notices of the Royal Astronomical Society: Letters, Volume 409, Issue 1, November 2010, Pages L64-L68, https://doi.org/10.1111/j.1745-3933.2010.00948.x

6. M. Zilberman. The "Dimming Effect" produced by the application of Doppler effect on the quantity of photons arriving to a receiver and its implication to astronomy. Intellectual Arch. 9, 3, 1-5 (2020), https://doi.org/10.32370/IA_2020_09_1

7. Deng Wang, Xin-He Meng. First comprehensive constraints on the Finslerian models using cosmological observations, Physics of the Dark Universe, Volume 21, 2018, Pages 55-60, ISSN 2212-6864, https://doi.org/10.1016/j.dark.2018.05.005.

8. A. Abramowski, F. Aharonian, F. Ait Benkhali, et all. Detailed spectral and morphological analysis of the shell type supernova remnant RCW 86, A\&A, Volume 612, April 2018. https://doi.org/10.1051/0004-6361/201526545 